\title{
Rate determining factors in protein model structures
}

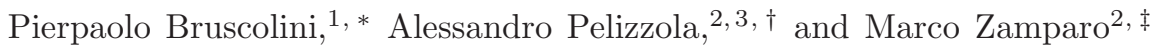 \\ ${ }^{1}$ Instituto de Biocomputación y Física de Sistemas Complejos (BIFI), \\ Universidad de Zaragoza, c. Corona de Aragón 42, 50009 Zaragoza, Spain \\ ${ }^{2}$ Dipartimento di Fisica and CNISM, Politecnico di Torino, c. Duca degli Abruzzi 24, 10129 Torino, Italy \\ ${ }^{3}$ INFN, Sezione di Torino
}

\begin{abstract}
Previous research has shown a strong correlation of protein folding rates to the native state geometry, yet a complete explanation for this dependence is still lacking. Here we study the rategeometry relationship with a simple statistical physics model, and focus on two classes of model geometries, representing ideal parallel and antiparallel structures. We find that the logarithm of the rate shows an almost perfect linear correlation with the "absolute contact order", but the slope depends on the particular class considered. We discuss these findings in the light of experimental results.
\end{abstract}

In the last decade many results have been published on the relationship between kinetic and structural features of protein folding, after the seminal work by Plaxco, Simons and Baker [1] revealed a simple linear relationship between the logarithm of the folding rate and the relative contact order (CO) for a set of two-state proteins. $\mathrm{CO}$ is defined as the sum of the sequence distances $|j-i|$ of any residue pair $(i, j)$ in contact in the native structure (with a "contact" being defined according to a cutoff distance), divided by the total number of contacts and number of residues. A similar good correlation was obtained by Jackson 2], considering the absolute contact order (ACO, equal to $\mathrm{CO}$ times the number of residues) and using a slightly extended protein set. Galzitskaya et al. in [3] showed that length, rather than CO, is relevant in three-state proteins. The role of the length is also considered in [4, 5, 6, 7], while the logarithm of the relative contact order, together with $\mathrm{CO}$ and $\mathrm{ACO}$, was considered in [8], and a combination of $\mathrm{CO}$ and length in [9]. The rates have been also related to linear combinations of the length and its logarithm [10], long range order (LRO), total contact distance [11, fraction of short range contacts [12], cliquishness 13], effective contact order 14]. Plotkin and coworkers [15] discussed the correlation with heterogeneities in contact distance and energy, while the absolute contact order, and its length dependence, was reconsidered in [16]. On the basis of the cited (and certainly not exhaustive) set of investigations it is difficult to reach a definite conclusion about which measures of native state properties are most relevant to determine the folding rate. Indeed, while experimental rates show a clear correlation with the structural parameters proposed in the above literature, they are always distributed with a relevant spread around the theoretical curve, that remains to be accounted for. On- and off-lattice theoretical modelling [17, 18] suggest that adding cooperativity and/or local preferential conformations to Gō like models improves the correlation of the rates with $\mathrm{CO}$, also inducing spread in the rate distribution. But also the high heterogeneity of the distribution of the sequence-distance of the contacts in protein structures could be responsi- ble for the rate spread [15]. For the above reasons, it is very important to try to address this issue with model structures, in the framework of a simple model with few parameters. We focus on two classes of structures, the ideal analogues of real parallel and antiparallel secondary structures $(\alpha$-helices and $\beta$-sheets): our aim is to find a set of rules that may be later used to rationalize the relationship between real protein geometries and rates.

We resort to the Wako-Saitô-Muñoz-Eaton (WSME) model, which allows an exact solution for the equilibrium thermodynamics and a very accurate semi-analytical approach to the kinetics. At difference with other nativecentric models, WSME is intrinsically cooperative and accounts for the local preferences of the main-chain. The model has already been shown to give good results in the determination of rates of real proteins [19, 20], their temperature dependence 21, the effect of mutations [22] and mechanical unfolding rates [23]. The WSME model was introduced in the end of the $70 \mathrm{~s}$ 24, 25] and then forgotten for roughly 20 years, when it was indepentently reproposed by Muñoz, Eaton and coworkers 19, 20, 26, 27]. Several studies followed [21, 23, 28, 29, 30, 31, 32, 33, 34, 35, 36] also with applications in a very different subfield of physics $37,38,39$. WSME is a Gō-like model [40], i.e. it is "native-centric", relying on the knowledge of the native state of a protein to describe its equilibrium and kinetics. Its binary degrees of freedom are related to the values of the dihedral angles at the peptide bonds [27], classified into just two states: ordered (native) and disordered (unfolded). Since the latter state allows a much larger number of microscopic realizations than the former, an entropic cost is given to the ordering of a peptide bond.

The model is described by the effective free energy:

$$
H=\sum_{i=1}^{N-1} \sum_{j=i+1}^{N} \epsilon_{i, j} \Delta_{i, j} \prod_{k=i}^{j} m_{k}-R T \sum_{k=1}^{N} q_{k}\left(1-m_{k}\right),
$$

where $N$ is the number of peptide bonds in the molecule, $R$ the ideal gas constant and $T$ the absolute temperature. $m_{k} \in\{0,1\}$ is the binary variable which tells whether the 
$k$-th bond, i.e. the one between residues $k$ and $k+1$, is in the disordered (0) or ordered (1) state, and $q_{k}$ is the corresponding ordering entropic cost. The product $\prod_{k=i}^{j} m_{k}$ takes value 1 if and only if all the peptide bonds from $i$ to $j$ are in the native state, thereby realizing the assumed interaction. The contact matrix with elements $\Delta_{i, j} \in\{0,1\}$ tells which peptide bonds are at close distance in the native state; non-native interactions are disregarded. The contact map beween peptide bonds, $\Delta_{i, j}$ is derived from the standard one between residues, $\Delta_{i, j}^{r}$, as $\Delta_{i, j}=\Delta_{i, j+1}^{r}$, thus assigning the (residue $i$ )-(residue $j$ ) contact to peptide bonds $i$ and $(j-1) . \Delta_{i, j}^{r}$ is usually calculated according to some cutoff distance residues $i$ and $j$ in the native state; here we deal with ideal secondary structures, and will impose $\Delta_{i, j}^{r}$ accordingly. Contact energy will be taken as $\epsilon_{i, j}=-\epsilon$ throughout the paper, unless differently stated. Without loss of generality, we can set $\epsilon=1$. Also the entropic costs will be taken to be homogeneous, $q_{k}=q=2 \ln 2$. Several values of these entropies have been considered in various works up to now, typically based on some fits to experimental data. Here we want to consider model structures, so the specific value is not too relevant: we take $q=2 \ln 2$, comparable to the results obtained for various molecules; in the following we will discuss the $q$-dependence of our results. Notice that, once fixed $\epsilon$ and $q$, the only source of heterogeneity comes from the contact matrix, i.e., from the geometry of the native state.

We define the denaturation temperature $T_{m}$ asking that the average fraction $m$ of ordered bonds is halfway between its values $m_{0}=1$ (at $\left.\mathrm{T}=0\right)$ and $m_{\infty}=$ $\frac{1}{N} \sum_{k=1}^{N}\left(1+e^{q_{k}}\right)^{-1}$ at infinite temperature. In the following, for each structure considered, we will always work at the corresponding $T_{m}$. Exact evaluation [24, 25] of thermodynamic quantities will be performed as in [34, 35].

The kinetic evolution of the model is described through a discrete-time master equation, $p_{t+1}(m)=$ $\sum_{m^{\prime}} W\left(m^{\prime} \rightarrow m\right) p_{t}(m)$, for the probability distribution $p_{t}(m)$ at time $t$, where $m=\left\{m_{k}, k=1, \ldots N\right\}$ denotes the state of the system. The transition matrix $W$ is specified by a single bond flip Metropolis rule, as in [21, 36]. The kinetics will be studied by means of the local equilibrium approach [21, 36], where the equilibration rate $k$ can be computed as the largest eigenvalue of a matrix of rank $N(N+1) / 2$. It has been shown in [21, 36] that this approach turns out to be very accurate when compared to exact or Monte Carlo results, and the rate so obtained is an upper bound of the exact one. Notably, this approach allows us to evaluate directly relaxation rates, which are the experimentally accessible quantities (at difference with folding and unfolding rates), without choosing a reaction coordinate.

We apply the model first to parallel structures (see Supplementary Material, Fig. 1), where the sequence distance of any interacting pair of residues is constant. This

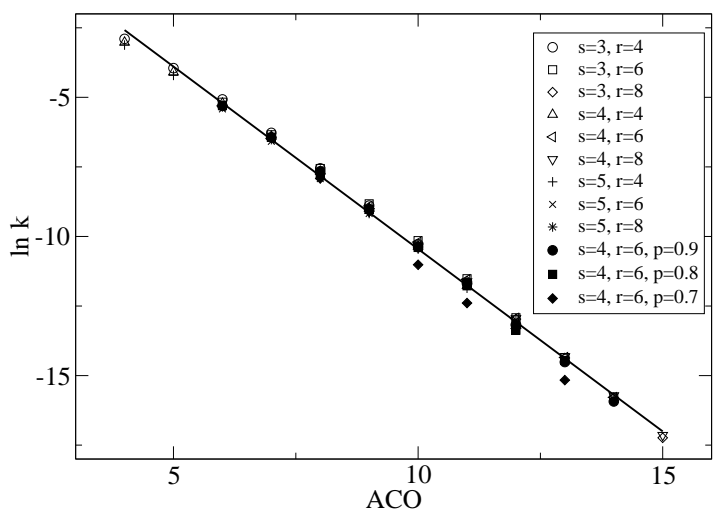

FIG. 1: Logarithm of equilibration rate vs. absolute contact order for parallel structures. Symbols explained in text.

class includes $\alpha$-helices and parallel $\beta$-sheets. The first structural indicator we consider is the ACO, defined as

$$
\mathrm{ACO}=N_{c}^{-1} \sum_{1 \leq i<j \leq N} \Delta_{i, j}(j-i+1),
$$

where $N_{c}=\sum_{1<i<j<N} \Delta_{i, j}$ is the total number of contacts, and we add 1 to the usual $j-i$, since our contact matrix is defined with reference to peptide bonds, and the number of the latter involved in a contact is the corresponding number of residues minus one.

In Fig. 1 we report the natural logarithm of the equilibration rate at $T_{m}$ for several parallel structures, defined as parallel $\beta$-sheets with $s$ strands and $r$ residues per strand, where consecutive strands are separated by loops of $l$ residues not involved in any contact. In such a structure the number of peptide bonds is $N=s(r+l)-l-1$ and $\mathrm{ACO}=r+l$; contact matrix elements are $\Delta_{i, j}=1$ if $j=i+l+r-1$ and $\Delta_{i, j}=0$ otherwise. The $\alpha$-helix corresponds to the case $l=0, \mathrm{ACO}=r=4$. For every $(s, r)$ pair we consider values of $l$ from 0 up to 8 in order to vary the ACO. The effect of dilution is also considered (for $s=4, r=6$ only), where this regular structure is perturbed by removing contacts with probability $1-p$. The value of $p$, when not otherwise specified, is 1 .

We see that all the results fall almost perfectly on the same straight line. A joint linear fit including all data yields $\ln k=2.6633-1.3113 \mathrm{ACO}$, with correlation coefficient -0.998. Considering different values for the entropic costs would not change the overall behaviour but only the slope. For instance, $q=2$ yields a slope -1.73 .

This result means that, according to this model, the $\mathrm{ACO}$ is of fundamental importance, while the rate cannot depend in a relevant way on other measures like the $\mathrm{CO}$ or the chain length, since each ACO value corresponds to several values of $\mathrm{CO}$ and total length. LRO, total contact distance, the fraction of short range contacts, and heterogeneity in contact distance are not applicable here, since all contacts present the same sequence distance. Even the introduction of contact-energy het- 

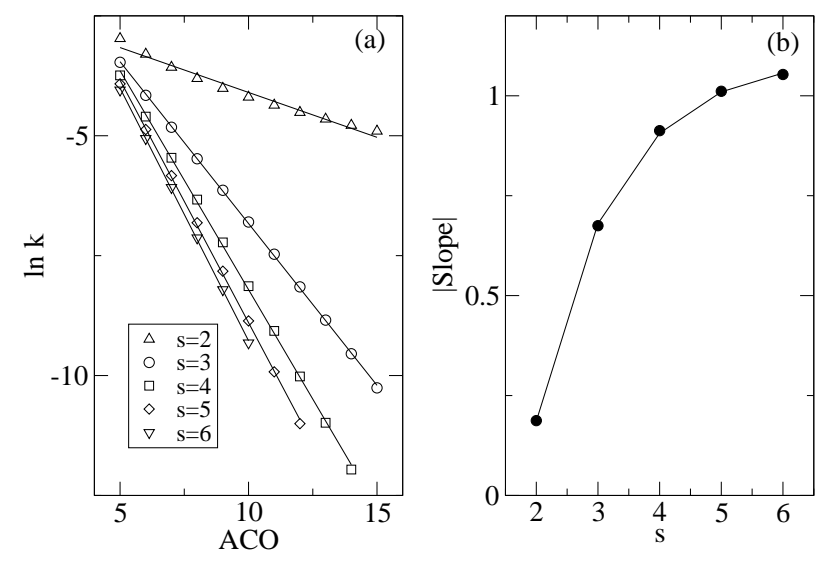

FIG. 2: (a) Logarithm of equilibration rate vs. absolute contact order for antiparallel structures at the denaturation temperature $T_{m}$. Symbols explained in text. (b) Absolute value of the slope in (a) as a function of $s$.

erogeneities does not affects the rate: considering $s=4$, $r=7$ and $l=0$ and 4 , and taking $\epsilon_{i, j}$ uniformly distributed in $[-\epsilon-\Delta \epsilon,-\epsilon+\Delta \epsilon]$, with $\Delta \epsilon \in[0, \epsilon]$, we found that the variation of $\ln k$ was compatible with the spread in Fig. 1. less than 0.02 in absolute value for $l=0$ ( $\ln k \simeq-5.45$ in the uniform case), and less than 0.6 for $l=4$ ( $\ln k \simeq-10.39$ in the uniform case). These figures hardly change if we consider the interactions as product of charges associated to residues, as in [41], and take random charges, or if we consider a correlated disorder, e.g. strenghtening the contacts of a group of three consecutive residues, leaving the others unchanged. In view of the above result it is worth checking whether this almost perfect linear behaviour still holds for structures which maintain some regularity, but where the contact distance is not constant. We therefore turn to antiparallel structures, and extend the results reported in 21] for a four-stranded antiparallel $\beta$-sheet, considering sheets with $s$ strands and $r$ residues per strand; in the case $s=2$ we have a simple hairpin (see Supplementary Material, Fig. 2). Turns corresponds to peptide bonds $k r$; contacts are established between bonds $i$ and $j=2 k r-i$, with $(k-1) r+1 \leq i \leq k r-1$ and $k=1, \cdots, s-1$ labelling the turns; the total number of peptide bonds is $N=s r-1$. This represents the most connected case: every residue but two (in the first and last turn) has at least one contact. The contact distance varies from 3 to $2 r-1$ in steps of 2 , and hence $\mathrm{ACO}=r+1$.

In Fig. 2(a) we report the natural logarithm of the equilibration rate at $T_{m}$ as a function of ACO for several $(s, r)$ values. It is clearly seen that the almost perfect linear correlation is now valid only at fixed strand number $s$ (again, slopes would be larger for a larger entropic cost $q$ ). The absolute value of the slope increases with $s$ and appears to tend to a constant for large $s$. Indeed, a fit to an exponential function is made in panel (b) and is al- most perfect $(\mid$ Slope $\mid=1.21-3.5 \exp (-0.62 s)$, estimated variance is 0.00017 , correlation coefficient is 0.9996$)$.

The limiting value of the slope is slightly smaller than that obtained for parallel structures, whence we have that, at fixed $\mathrm{ACO} \gtrsim 4$, the hairpin is the fastest structure, then the other antiparallel structures come, in order of increasing $s$, and finally we have the parallel structures.

The exponential dependence of the logarithm of the rate on $s$ is quite puzzling: to understand such a behavior, we study the folding mechanism, as revealed by the probabilities of native "strings" $S_{i, j}$, i.e., of configurations where peptide bonds $i$ and $j$ are unfolded, while all those in between them are native. Assuming that the relaxation rate can be related to the height of the highest barrier along the folding pathway, the analysis reveals that the latter is represented by the folding of one hairpin, or more precisely of all the residues between peptide bond $u=(k-1) r$ and $v=(k+1) r+1$, being $u$ and $v$ unfolded, for any $k$ between 1 and $(s-1)$. The probability of such a configuration can be estimated as $p\left(S_{u, v}\right)=\exp \left(\beta_{m} \epsilon(r-1)-2 r q\right) / Z_{u, v}$, where $\beta_{m}=1 /\left(R T_{m}\right) . Z_{u, v}$ is the partition function restricted to the region between $u$ and $v$. It can be estimated as

$$
\begin{aligned}
Z_{u, v} & =A^{2 r-1}+A e^{\beta_{m} \epsilon(r-1)-(2 r-1) q} \\
& +\left(2 e^{-q}+1\right) \sum_{j=0}^{r-2} A^{2(r-j)-3} e^{\beta_{m} \epsilon j-(2 j+1) q},
\end{aligned}
$$

where $A=(1+\exp (-q))$. If our system can be considered a two-state folder we have that the relaxation rate $k=k_{f}+k_{u}$ is the sum of the folding and unfolding rates; the latter will be identical at $T=T_{m}$ and, assuming an Arrhenius scheme, they will be proportional to the folding probability $p\left(S_{u, v}\right)$. So, if the above assumptions hold and we have identified the correct barrier, we will find the same behavior for $\ln p\left(S_{u, v}\right)$ as for $\ln k$. Notice that the expression of $p\left(S_{u, v}\right)$ contains clearly the ACO, since $r=\mathrm{ACO}-1$, while the only quantity depending on $s$ is the value of $\beta_{m}$ : one finds indeed that the exponential dependence of $\ln k$ on $s$ is related to the dependence of $\beta_{m}$ on s and ACO. Taking this into account in the expression of $p\left(S_{u, v}\right)$ yields a dependence on ACO which is completely analogous to that reported in Fig. 22, where again $\ln p\left(S_{u, v}\right)$ can be fitted by straight lines whose slopes follow the law: $\mid$ Slope $\mid=1.40-5.1 \exp (-0.70 s)$, (estimated variance: 0.0012 , correlation coefficient: 0.9980$)$ reasonably close to the value obtained for the rates.

The above result is very interesting, because it proves that an Arrhenius framework can still be applied in this case, despite the free-energy profiles may present more than one minimum and barrier. But even more, these results establish a quantitative connection between the rate and the number of strands through the stability, stating that as the number of strands of the system increases, the $T_{m}$ increases asymptotically, implying a global increase of the stability, while the equilibration rate decreases. 
Notice that the dependence of the rate on $s$ found in the model is consistent with the reported observation of a $L^{\alpha}$ - dependence [16]: indeed if we fit the rate to the number of residues $L=s r$ we find for the antiparallel structures $\ln k=-0.73 \times L^{0.64}$, which is not far from the experimental exponent reported in $[16](\alpha=0.70)$ and from $\alpha=0.61$ coming from off-lattice simulations [9]. However, an accurate comparison of the dependence of rates on length should take into account the frequency of the different structures in real proteins, which is out of the scope of the present work.

To conclude, let us review the main findings: resorting to a simple statistical model, we have performed a detailed analysis of the dependence of the relaxation rate on some structural indicators, known to correlate with protein folding times. To elucidate the role and the interplay of the different factors, we have studied ideal helical, parallel and antiparallel secondary structures, of different length and number of strands. Our results confirm the absolute contact order $\mathrm{ACO}$ as the main structural determinant of the rates, but suggest different folding mechanisms for parallel and antiparallel structures: the latter, which fold faster than the former at equal ACO, show a dependence on the number of strands which we can relate to the dependence of the denaturation temperature on the ACO and number of strands. We also find that the dependence of the rate on the length of the protein is consistent with the power-law reported in [16] for real proteins, despite the fact our ideal structures lack the structural and energetic heterogeneity of the latter.

P. B. acknowledges support from Spanish Education and Science Ministry (FIS2004-05073, FIS2006-12781).

* Electronic address: pier@unizar.es

† Electronic address: alessandro.pelizzola@polito.it

¥ Electronic address: marco.zamparo@polito.it

[1] K. W. Plaxco, K. T. Simons and D. Baker, J. Mol. Biol. 277, 985 (1998).

[2] S. E. Jackson, Fold. Des. 3, R81 (1998).

[3] O. V. Galzitskaya,S. O. Garbuzynskiy, D. N. Ivankov, and A. V. Finkelstein, Proteins: Struct. Funct. Genet. $\mathbf{5 1}, 162$ (2003).

[4] D. Thirumalai, J. Phys. I (France) 5, 1457 (1995).

[5] M. Cieplak and T.X. Hoang, Biophys. J. 84, 475 (2003).

[6] M. S. Li, D. K. Klimov, andD. Thirumalai, Polymer 45, 573 (2004).

[7] A. N. Naganathan and V. Muñoz, J. Am. Chem. Soc. 127, 480 (2005).

[8] V. Grantcharova, E. J. Alm, D. Baker and A. L. Horwich, Curr. Opin. Struct. Biol. 11, 70 (2001).

[9] N. Koga and S. Takada, J. Mol. Biol. 313, 171 (2001).
[10] D.E. Makarov, C.A. Keller, K.W. Plaxco and H. Metiu, Proc. Natl. Acad. Sci. U.S.A. 99, 3535 (2002).

[11] H. Zhou and Y. Zhou, Biophys. J. 82, 458 (2002).

[12] E.J. Miller, K.F. Fischer and S. Marqusee, Proc. Natl. Acad. Sci. U.S.A. 99, 10359 (2002).

[13] C. Micheletti, Proteins: Struct. Funct. Genet. 51, 74 (2003).

[14] P.D. Dixit and T.R. Weikl, Proteins: Struct. Funct. Bioinf. 64, 193 (2006).

[15] B. Ötzop, M.R. Ejtehadi and S.S. Plotkin, Phys. Rev. Lett. 93, 208105 (2004).

[16] D. N. Ivankov, S. O. Garbuzynskiy, E. Alm, K. W. Plaxco,D. Baker, and A. V. Finkelstein, Protein Sci. 12, 2057 (2003).

[17] A. I. Jewett,V. S. Pande, and K. W. Plaxco, J. Mol. Biol. 326, 247 (2003).

[18] H. Kaya and H. S. Chan, Proteins: Struct. Funct. Genet,,52, 524 (2003).

[19] V. Muñoz and W.A. Eaton, Proc. Natl. Acad. Sci. U.S.A. 96, 11311 (1999).

[20] E.R. Henry, W.A. Eaton, Chem. Phys. 307, 163 (2004).

[21] M. Zamparo and A. Pelizzola, Phys. Rev. Lett. 97, 068106 (2006).

[22] A. Pelizzola and M. Zamparo, in preparation.

[23] A. Imparato, A. Pelizzola and M. Zamparo, Phys. Rev. Lett. 98, 148102 (2007).

[24] H. Wako and N. Saitô, J. Phys. Soc. Jpn 44, 1931 (1978).

[25] H. Wako and N. Saitô, J. Phys. Soc. Jpn 44, 1939 (1978).

[26] V. Muñoz, P.A. Thompson, J. Hofrichter and W.A. Eaton, Nature 390, 196 (1997).

[27] V. Muñoz, E.R. Henry, J. Hofrichter and W.A. Eaton, Proc. Natl. Acad. Sci. U.S.A. 95, 5872 (1998).

[28] A. Flammini, J.R. Banavar and A. Maritan, Europhys. Lett. 58, 623 (2002).

[29] I. Chang, M. Cieplak, J.R. Banavar and A. Maritan, Protein Sci. 13, 2446 (2004).

[30] K. Itoh and M. Sasai, Proc. Natl. Acad. Sci. U.S.A. 101, 14736 (2004).

[31] K. Itoh and M. Sasai, Chem. Phys. 307, 121 (2004).

[32] K. Itoh and M. Sasai, Proc. Natl. Acad. Sci. U.S.A. 103, 7298 (2006).

[33] H. Abe and H. Wako, Phys. Rev. E 74, 011913 (2006).

[34] P. Bruscolini and A. Pelizzola, Phys. Rev. Lett. 88, 258101 (2002).

[35] A. Pelizzola, J. Stat. Mech., P11010 (2005).

[36] M. Zamparo and A. Pelizzola, J. Stat. Mech., P12009 (2006).

[37] V. I. Tokar and H. Dreyssé, Phys. Rev. E 68, 011601 (2003).

[38] V. I. Tokar and H. Dreyssé, J. Phys. Cond. Matter 16, S2203 (2004).

[39] V. I. Tokar and H. Dreyssé, Phys. Rev. E 71, 031604 (2005).

[40] Y. Udea, H. Taketomi, N. Gō, Biopolymers 17, 1531 (1978).

[41] G. Tiana, F. Simona, G. M. S. De Mori, R. A. Broglia and G. Colombo, Prot. Sci. 13, 113 (2004). 